\title{
Generalized Induced Norms ${ }^{⿴ 囗 十}$
}

\author{
S. Hejazian, M. Mirzavaziri and M. S. Moslehian
}

\begin{abstract}
Let $\|$.$\| be a norm on the algebra M_{n}$ of all $n \times n$ matrices over $\mathbb{C}$. An interesting problem in matrix theory is that "are there two norms $\|\cdot\|_{1}$ and $\|\cdot\|_{2}$ on $\mathbb{C}^{n}$ such that $\|A\|=\max \left\{\|A x\|_{2}:\|x\|_{1}=1\right\}$ for all $A \in M_{n}$. We will investigate this problem and its various aspects and will discuss under which conditions $\|\cdot\|_{1}=\|\cdot\|_{2}$.
\end{abstract}

\section{Preliminaries}

Throughout the paper $M_{n}$ denotes the complex algebra of all $n \times n$ matrices $A=\left[a_{i j}\right]$ with entries in $\mathbb{C}$ together with the usual matrix operations. Denote by $\left\{e_{1}, e_{2}, \cdots e_{n}\right\}$ the standard basis for $\mathbb{C}^{n}$, where $e_{i}$ has 1 as its $i$ th entry and 0 elsewhere. We denote by $E_{i j}$ the $n \times n$ matrix with 1 in the $(i, j)$ entry and 0 elsewhere.

For $1 \leq p \leq \infty$ the norm $\ell_{p}$ on $\mathbb{C}^{n}$ is defined as follows:

$$
\ell_{p}(x)=\ell_{p}\left(\sum_{i=1}^{n} x_{i} e_{i}\right)=\left\{\begin{array}{cc}
\left(\sum_{i=1}^{n}\left|x_{i}\right|^{p}\right)^{1 / p} & 1 \leq p<\infty \\
\max \left\{\left|x_{1}\right|, \cdots,\left|x_{n}\right|\right\} & p=\infty
\end{array}\right.
$$

A norm $\|$.$\| on \mathbb{C}^{n}$ is said to be unitarily invariant if $\|x\|=\|U x\|$ for all unitaries $U$ and all $x \in \mathbb{C}^{n}$.

*2000 Mathematics Subject Classification 15A60 (Primary) 47A30, 46B99 (Secondary).

Keywords and phrases. induced norm, generalized induced norm, algebra norm, the full matrix algebra, unitarily invariant, generalized induced congruent. 
By an algebra norm (or a matrix norm) we mean a norm $\|\cdot\|$ on $M_{n}$ such that $\|A B\| \leq$ $\|A\|\|B\|$ for all $A, B \in M_{n}$. An algebra norm $\|$.$\| on M_{n}$ is called unitarily invariant if $\|U A V\|=\|A\|$ for all unitaries $U$ and $V$ and all $A \in M_{n}$. See [2, Chapter IV] for more information.

Example 1.1 The norm $\|A\|_{\sigma}=\sum_{i, j=1}^{n}\left|a_{i j}\right|$ is an algebra norm, but the norm $\|A\|_{m}=$ $\max \left\{\left|a_{i, j}\right|: 1 \leq i, j \leq n\right\}$ is not an algebra norm, since $\left\|\left[\begin{array}{ll}1 & 1 \\ 1 & 1\end{array}\right]^{2}\right\|_{m}>\left\|\left[\begin{array}{ll}1 & 1 \\ 1 & 1\end{array}\right]\right\|_{m}^{2}$.

Remark 1.2 It is easy to show that for each norm $\|$.$\| on M_{n}$, the scaled norm $\max \left\{\frac{\|A B\|}{\|A\|\|B\|}\right.$ : $A, B \neq 0\}\|$.$\| is an algebra norm; cf. [1, p.114]$

Let $\|\cdot\|_{1}$ and $\|\cdot\|_{2}$ be two norms on $\mathbb{C}^{n}$. Then for each $A:\left(\mathbb{C}^{n},\|\cdot\|_{1}\right) \rightarrow\left(\mathbb{C}^{n},\|\cdot\|_{2}\right)$ we can define $\|A\|=\max \left\{\|A x\|_{2}:\|x\|_{1}=1\right\}$. If $\|\cdot\|_{1}=\|\cdot\|_{2}$, then $\|I\|=1$ and there are many examples of $\|\cdot\|_{1}$ and $\|\cdot\|_{2}$ such that $\|I\| \neq 1$. This shows that given $\|\cdot\|$ on $M_{n}$, we cannot deduce in general that there is a norm $\|\cdot\|_{1}$ on $\mathbb{C}^{n}$ with $\|A\|=\max \left\{\|A x\|_{1}:\|x\|_{1}=1\right\}$. Let us recall the concept of g-ind norm as follows:

Definition 1.3 Let $\|\cdot\|_{1}$ and $\|\cdot\|_{2}$ be two norms on $\mathbb{C}^{n}$. Then the norm $\|\cdot\|_{1,2}$ on $M_{n}$ defined by $\|A\|_{1,2}=\max \left\{\|A x\|_{2}:\|x\|_{1}=1\right\}$ is called the generalized induced (or g-ind) norm via $\|\cdot\|_{1}$ and $\|\cdot\|_{2}$. If $\|\cdot\|_{1}=\|\cdot\|_{2}$, then $\|\cdot\|_{1,1}$ is called induced norm.

Example 1.4 $\|A\|_{C}=\max \left\{\sum_{i=1}^{n}\left|a_{i, j}\right|: 1 \leq j \leq n\right\},\|A\|_{R}=\max \left\{\sum_{j=1}^{n}\left|a_{i, j}\right|: 1 \leq i \leq n\right\}$ and the spectral norm $\|A\|_{S}=\max \left\{\sqrt{\lambda}: \lambda\right.$ is an eigenvalue of $\left.A^{*} A\right\}$ are induced by $\ell_{1}, \ell_{\infty}$ and $\ell_{2}$ (or the Eucleadian norm), respectively.

It is known that the algebra norm $\|A\|=\max \left\{\|A\|_{C},\|A\|_{R}\right\}$ is not induced [ ] and it is not hard to show that it is not g-ind too; cf. [1, Corollary 3.2.6]

We need the following proposition which is a special case of a finite dimensional version of the Hahn-Banach theorem [5, p. 104]: 
Proposition 1.5 Let $\|$.$\| be a norm on \mathbb{C}^{n}$ and $y \in \mathbb{C}^{n}$ be a given vector. There exists a vector $y_{\circ} \in \mathbb{C}^{n}$ such that $y_{\circ}^{*} y=\|y\|$ and for all $x \in \mathbb{C}^{n},\left|y_{\circ}^{*} x\right| \leq\|x\|$. (Throughout, $*$ denotes the transpose) [3, Corollary 5.5.15])

In this paper we examine the following nice problems:

(i) Given a norm $\|$.$\| on M_{n}$ is there any class $\mathcal{A}$ of $M_{n}$ such that the restriction of the norm $\|$.$\| on \mathcal{A}$ is g-ind?

(ii) When a g-ind norm is unitarily invariant?

(iii) If a given norm $\|\cdot\|$ is g-ind via $\|\cdot\|_{1}$ and $\|\cdot\|_{2}$, then is it possible to find $\|\cdot\|_{1}$ and $\|\cdot\|_{2}$ explicitly in terms of $\|$.$\| ?$

(iv) When two g-ind norms are the same?

(v) Is there any characterization of the g-ind norms which are algebra norms?

\section{Main Results}

We begin with some observations on generalized induced norms.

Let $\|\cdot\|_{1,2}$ be a generalized induced norm on $M_{n}$ obtained via $\|\cdot\|_{1}$ and $\|\cdot\|_{2}$. Then $\left\|E_{i j}\right\|_{1,2}=\max \left\{\left\|E_{i j} x\right\|_{2}:\|x\|_{1}=1\right\}=\max \left\{\left\|x_{j} e_{i}\right\|_{2}:\left\|\left(x_{1}, \cdots, x_{j}, \cdots, x_{n}\right)\right\|_{1}=1\right\}=$ $\alpha_{j}\left\|e_{i}\right\|_{2}$, where $\alpha_{j}=\max \left\{\left|x_{j}\right|:\left\|\left(x_{1}, \cdots, x_{j}, \cdots, x_{n}\right)\right\|_{1}=1\right\}$. In general, for $x \in \mathbb{C}^{n}$ and $1 \leq j \leq n$, if $C_{x, j} \in M_{n}$ is defined by the operator $C_{x, j}(y)=y_{j} x$ then $\left\|C_{x, j}\right\|_{1,2}=\alpha_{j}\|x\|_{2}$.

Also if for $x \in \mathbb{C}^{n}$ we define $C_{x} \in M_{n}$ by $C_{x}=\sum_{j=1}^{n} C_{x, j}$, then clearly $\left\|C_{x}\right\|_{1,2}=\alpha\|x\|_{2}$, where $\alpha=\max \left\{\left|\sum_{j=1}^{n} y_{j}\right|:\left\|\left(y_{1}, \cdots, y_{j}, \cdots, y_{n}\right)\right\|_{1}=1\right\}$.

Now we give a partial solution to Problem (i) and useful direction toward solving Problem (iii):

Proposition 2.1 Let $\|$.$\| be an algebra norm on M_{n}$. Then $\|$.$\| is a g-ind norm on \{A \in$ $\left.M_{n}:\|A\|=\left\|A^{-1}\right\|=1\right\}$.

Proof. Put $\|x\|_{1}=\max \left\{\left\|C_{A x}\right\|:\|A\|=1\right\}, \lambda^{-1}=\max \left\{\left|\sum_{i=1}^{n} x_{i}\right|:\|x\|_{1}=1\right\}$ and $\|x\|_{2}=$ $\lambda\left\|C_{x}\right\|$. 
Then we have $\left\|C_{y}\right\|_{1,2}=\max \left\{\left\|C_{y} x\right\|_{2}:\|x\|_{1}=1\right\}=\max \left\{\left|\sum_{i=1}^{n} x_{i}\right|\|y\|_{2}:\|x\|_{1}=1\right\}=$ $\|y\|_{2} \lambda^{-1}=\left\|C_{y}\right\|$.

It follows that for each $y \in \mathbb{C}^{n}$ there is some $x \in \mathbb{C}^{n}$ such that $\left\|C_{y} x\right\|_{2}=\left\|C_{y}\right\|\|x\|_{1}=$ $\left\|C_{y}\right\| \max \left\{\left\|C_{D x}\right\|:\|D\|=1\right\}$.

Now let $A$ be invertible and $\left\|A^{-1}\right\|=\|A\|=1$ and $z=A^{-1} C_{y} x$. Then $\lambda^{-1}\|B z\|_{2}=$ $\lambda^{-1}\left\|B A^{-1} C_{y} x\right\|_{2}=\lambda^{-1}\|D x\|_{2}=\left\|C_{D x}\right\| \leq \frac{1}{\left\|C_{y}\right\|}\left\|C_{y} x\right\|_{2}=\frac{1}{\left\|C_{y}\right\|}\|A z\|_{2}$.

Now choose $y$ so that $\left\|C_{y}\right\|=1$. Then $\left\|C_{B z}\right\| \leq\left\|C_{A z}\right\|$ for all $B \in M_{n}$. This implies that $\left\|C_{A z}\right\|$ is an upper bound for the set $\left\{\left\|C_{B z}\right\|:\|B\|=1\right\}$ and indeed $\left\|C_{A z}\right\|=\max \left\{\left\|C_{B z}\right\|\right.$ : $\|B\|=1\}=\|z\|_{1}$. It follows that $\|A\|=1=\left\|C_{A\left(\frac{z}{\|z\|_{1}}\right)}\right\|=\max \left\{\left\|C_{A u}\right\|:\|u\|_{1}=1\right\}=$ $\max \left\{\|A u\|_{2}:\|u\|_{1}=1\right\}=\|A\|_{1,2} \cdot \square$

Let us now answer Question (ii).

Proposition 2.2 An induced norm $\|\cdot\|_{1,2}$ is unitarily invariant if and only if so are $\|\cdot\|_{1}$ and $\|\cdot\|_{2}$.

Proof. Let $U, V$ be unital operators and $A$ be an arbitrary operator on $\mathbb{C}^{n}$. Suppose that $\|\cdot\|_{1}$ and $\|\cdot\|_{2}$ are unitarily invariant. Then

$$
\|U A V\|_{1,2}=\max _{x \neq 0} \frac{\|U A V x\|_{2}}{\|x\|_{1}}=\max _{x \neq 0} \frac{\|A V x\|_{2}}{\|x\|_{1}}=\max _{y \neq 0} \frac{\|A y\|_{2}}{\left\|V^{-1} x\right\|_{1}}=\max _{y \neq 0} \frac{\|A y\|_{2}}{\|y\|_{1}}=\| A_{1,2} .
$$

Conversely, if $\|\cdot\|_{1,2}$ is unitarily invariant, then $\|U x\|_{1}=\max \left\{\|A U x\|_{2}:\|A\|_{1,2} \leq 1\right\}=$ $\max \left\{\|B x\|_{2}:\left\|U^{-1} B\right\|_{1,2} \leq 1\right\}=\max \left\{\|B x\|_{2}:\|B\|_{1,2} \leq 1\right\}=\|x\|_{1}$ and $\|U x\|_{2}=\frac{1}{\alpha}\left\|C_{U x}\right\|=$ $\frac{1}{\alpha}\left\|U C_{x}\right\|=\frac{1}{\alpha}\left\|U C_{x}\right\|=\frac{1}{\alpha}\left\|C_{x}\right\|=\|x\|_{2}$.

Modifying the proof of Theorem 5.6.18 of [3], we obtain a similar useful result for g-ind norms:

Theorem 2.3 Let $\|\cdot\|_{1},\|\cdot\|_{2},\|\cdot\|_{3}$ and $\|\cdot\|_{4}$ be four given norms on $\mathbb{C}^{n}$ and

$$
R_{i, j}=\max \left\{\frac{\|x\|_{i}}{\|x\|_{j}}: x \neq 0\right\}, 1 \leq i, j \leq 4 .
$$

Then

$$
\max \left\{\frac{\|A\|_{1,2}}{\|A\|_{3,4}}: A \neq 0\right\}=R_{2,4} R_{3,1}
$$


In particual, $\max \left\{\frac{\|A\|_{1,1}}{\|A\|_{2,2}}: A \neq 0\right\}=\max \left\{\frac{\|A\|_{2,2}}{\|A\|_{1,1}}: A \neq 0\right\}=R_{1,2} R_{2,1}$.

Proof. Let $A$ be a matrix and $x \neq 0$. Then $\frac{\|A x\|_{2}}{\|x\|_{1}}=\frac{\|A x\|_{2}}{\|A x\|_{4}} \cdot \frac{\|A x\|_{4}}{\|x\|_{3}} \cdot \frac{\|x\|_{3}}{\|x\|_{1}}$. Hence $\|A\|_{1,2} \leq$ $R_{2,4}\|A\|_{3,4} R_{3,1}$. Thus $\frac{\|A\|_{1,2}}{\|A\|_{3,4}} \leq R_{2,4} R_{3,1}$.

There are vectors $y, z$ in $\mathbb{C}^{n}$ such that $\|y\|_{2}=\|z\|_{2}=1,\|y\|_{2}=R_{2,4}\|y\|_{4}$ and $\|z\|_{3}=$ $R_{3,1}\|z\|_{1}$. By Proposition 1.15, there exists a vector $z_{\circ} \in \mathbb{C}^{n}$ such that $\left|z_{\circ}^{*} x\right| \leq\|x\|_{3}$ and $z_{0}^{*} z=\|z\|_{3}$.

Put $A_{\circ}=y z_{\circ}$. Then $\frac{\left\|A_{\circ} z\right\|_{2}}{\|z\|_{1}}=\frac{\left\|y z_{z}^{*} z\right\|_{2}}{\|z\|_{1}}=\frac{\|y\|_{2}\|z\|_{3}}{\|z\|_{1}}=\|y\|_{2} R_{3,1}$. Hence $\left\|A_{\circ}\right\|_{1,2} \geq \frac{\|y\|_{2}}{\|y\|_{4}} R_{3,1}\|y\|_{4}=$ $R_{2,4} \cdot R_{3,1}\|y\|_{4}$. On the other hand, $\frac{\left\|A_{\circ} x\right\|_{4}}{\|x\|_{3}}=\frac{\left\|y z_{\circ}^{*} x\right\|_{4}}{\|x\|_{3}}=\frac{\|y\|_{4} z_{\circ}^{*} x \mid}{\|x\|_{3}} \leq\|y\|_{4}$. Thus $\left\|A_{\circ}\right\|_{3,4} \leq\|y\|_{4}$. Hence $\frac{\left\|A_{\circ}\right\|_{1,2}}{\left\|A_{\circ}\right\|_{3,4}} \geq \frac{R_{2,4} R_{3,1}\|y\|_{4}}{\|y\|_{4}}=R_{2,4} R_{3,1}$.

Corollary 2.4 (i) $\|\cdot\|_{1,2} \leq\|\cdot\|_{3,2}$ if and only if $\|\cdot\|_{1} \geq\|\cdot\|_{3}$,

(ii) $\|\cdot\|_{1,2} \leq\|\cdot\|_{1,4}$ if and only if $\|\cdot\|_{2} \leq\|\cdot\|_{4}$.

Proof. (i) $\|\cdot\|_{1,2} \leq\|\cdot\|_{3,2}$ if and only if $\max \left\{\frac{\|A\|_{1,2}}{\|A\|_{3,2}}: A \neq 0\right\}=R_{2,2} R_{3,1} \leq 1$ and this if and only if $R_{3,1} \leq 1$ or equivalently $\|\cdot\|_{3} \leq\|\cdot\|_{1}$. The proof of (ii) is similar. $\square$

The following corollary completely answers to Question (iv):

Corollary $2.5\|\cdot\|_{1,2}=\|\cdot\|_{3,4}$ if and only if there exists $\gamma>0$ such that $\|\cdot\|_{1}=\gamma\|\cdot\|_{3}$ and $\|\cdot\|_{2}=\gamma\|\cdot\|_{4}$.

Proof. If $\|A\|_{1,2}=\|A\|_{3,4}$, then $R_{4,2} R_{1,3}=\max \left\{\frac{\|A\|_{3,4}}{\|A\|_{1,2}}: A \neq 0\right\}=1=\max \left\{\frac{\|A\|_{1,2}}{\|A\|_{3,4}}: A \neq\right.$ $0\}=R_{2,4} R_{3,1}$. Hence $\max \left\{\frac{\|x\|_{2}}{\|x\|_{4}}: x \neq 0\right\}=R_{2,4}=\frac{1}{R_{3,1}}=\min \left\{\frac{\|x\|_{1}}{\|x\|_{3}}: x \neq 0\right\} \leq \max \left\{\frac{\|x\|_{1}}{\|x\|_{3}}:\right.$ $x \neq 0\}=R_{1,3}=\frac{1}{R_{4,2}}=\min \left\{\frac{\|x\|_{2}}{\|x\|_{4}}: x \neq 0\right\}$. Thus there exists a number $\gamma$ such that $\frac{\|x\|_{2}}{\|x\|_{4}}=\gamma=\frac{\|x\|_{1}}{\|x\|_{3}}$.

Remark 2.6 It is known that each induced norm $\|$.$\| is minimal in the sense that for any$ matrix norm $\|\cdot\|$, the inequality $\|\cdot\| \leq\|\cdot\|_{1,1}$ implies that $\|\cdot\|=\|\cdot\|_{1,1}$. But this is not true for g-ind norms in general. For instance, put $\|.\|_{\alpha}=\ell_{\infty}(),.\|\cdot\|_{\beta}=2 \ell_{2}($.$) and \|\cdot\|_{\gamma}=\ell_{2}($.$) .$ Then $\|\cdot\|_{\gamma, \beta} \leq\|\cdot\|_{\alpha, \beta}$ but $\|\cdot\|_{\gamma, \beta} \neq\|\cdot\|_{\alpha, \beta}$. 
The following theorem is one of our main theorems and provide a complete solution for Problem (v):

Theorem 2.7 Let $\|\cdot\|_{1}$ and $\|\cdot\|_{2}$ be two norms on $\mathbb{C}^{n}$. Then $\|\cdot\|_{1,2}$ is an algebra norm on $M_{n}$ if and only if $\|\cdot\|_{1} \leq\|\cdot\|_{2}$.

Proof. For each $A$ and $B$ in $M_{n}$ we have

$$
\|A B x\|_{2} \leq\|A\|_{1,2}\|B x\|_{1} \leq\|A\|_{1,2}\|B x\|_{2} \leq\|A\|_{1,2}\|B\|_{1,2}\|x\|_{1} .
$$

Hence $\|A B\|_{1,2} \leq\|A\|_{1,2}\|B\|_{1,2}$.

Conversely, let $\|\cdot\|_{1,2}$ be an algebra norm. Then for each $A, B \in M_{n}$ we have $\|A B\|_{2} \leq$ $\|A\|_{1,2}\|B\|_{1,2}\|x\|_{1}$. Let $B$ be an arbitrary member of $M_{n}$. For $B x \neq 0$, take $M$ to be the linear span of $\{B x\}$ and define $f:\left(M,\|\cdot\|_{1}\right) \rightarrow \mathbb{C}$ by $f(c B x)=\frac{c\|B\|_{1}}{\|B x\|_{2}}$. By the Hahn-Banach Theorem, there is an $F:\left(\mathbb{C}^{n},\|\cdot\|_{1}\right) \rightarrow \mathbb{C}$ with $\left.F\right|_{M}=f$ and $\|F\|=\|f\|=\max \{|f(c B x)|$ : $\left.\|c B x\|_{1}=1\right\}=\max \left\{\frac{|c|\|B x\|_{1}}{\|B x\|_{2}}:|c|\|B x\|_{1}=1\right\}=\frac{1}{\|B x\|_{2}}$. Define $A:\left(\mathbb{C}^{n},\|\cdot\|_{1}\right) \rightarrow\left(\mathbb{C}^{n},\|\cdot\|_{2}\right)$ by $A y=F(y) B x$. Then $\|A\|_{1,2}=\max \left\{\|A y\|_{2}:\|y\|_{1}=1\right\}=\max \left\{|F(y)|\|B x\|_{2}:\|y\|_{1}=1\right\}=$ 1 , and $\|A B x\|_{2}=|F(B x)|\|B x\|_{2}=|f(B x)|\|B x\|_{2}=\frac{\|B x\|_{1}}{\|B x\|_{2}}\|B x\|_{2}=\|B x\|_{1}$. Thus for all $B$,

$$
\|B x\|_{1}=\|A B x\|_{2} \leq\|A\|_{1,2}\|B\|_{1,2}\|x\|_{1}=\|B\|_{1,2}\|x\|_{1},
$$

or

$$
\|B x\|_{1} \leq\|B\|_{1,2}\|x\|_{1} .
$$

Now take $N$ to be the linear span of $\{x\}$ and define $g:\left(N,\|\cdot\|_{1}\right) \rightarrow \mathbb{C}$ by $g(c x)=\frac{c\|x\|_{1}}{\|x\|_{2}}$. By the Hahn-Banach Theorem, there is a $G:\left(\mathbb{C}^{n},\|\cdot\|_{1}\right) \rightarrow \mathbb{C}$ with $\left.G\right|_{N}=g$ and $\|G\|=\|g\|=$ $\max \left\{|g(c x)|:\|c x\|_{1}\right\}=\max \left\{\frac{|c|\|x\|_{1}}{\|x\|_{2}}:|c|\|x\|_{1}=1\right\}=\frac{1}{\|x\|_{2}}$. Define $B:\left(\mathbb{C}^{n},\|\cdot\|_{1}\right) \rightarrow\left(\mathbb{C}^{n},\|\cdot\|_{2}\right)$ by $B y=G(y) x$. Then $\|B\|_{1,2}=\max \left\{\|B y\|_{2}:\|y\|_{1}=1\right\}=\max \left\{|G(y)|\|x\|_{2}:\|y\|_{1}=1\right\}=$ $\|x\|_{2}\|G\|=1$, and $\|B x\|_{1}=|G(x)|\|x\|_{1}=|g(x)|\|x\|_{1}=\frac{\|x\|_{1}}{\|x\|_{2}}\|x\|_{1}=\frac{\|x\|_{1}^{2}}{\|x\|_{2}}$.

So

$$
\frac{\|x\|_{1}^{2}}{\|x\|_{2}}=\|B x\|_{1} \leq\|B\|_{1,2}\|x\|_{1}=\|x\|_{1}
$$

Thus $\|.\|_{1} \leq\|\cdot\|_{2}$. 
Proposition 2.8 Suppose that $\|\cdot\|_{1,2}$ is a g-ind norm and $\lambda>0$. Then the scaled norm $\lambda\|\cdot\|_{1,2}$ is a g-ind algebra norm if and only if $\lambda \geq R_{1,2}$.

Proof. Evidently, $\lambda\|\cdot\|_{1,2}=\|\cdot\|_{\|\cdot\|_{1}, \lambda\|\cdot\|_{2}}$. If $\|\cdot\|_{3,4}=\lambda\|\cdot\|_{1,2}=\|\cdot\|_{\|\cdot\|_{1}, \lambda\|\cdot\|_{2}}$ then Corollary 2.5 implies that there exists $\alpha>0$ such that $\|\cdot\|_{3}=\alpha\|\cdot\|_{1}$ and $\|\cdot\|_{4}=\alpha \lambda\|\cdot\|_{2}$. Now Theorem 2.7 follows that $\lambda\|\cdot\|_{1,2}=\|\cdot\|_{3,4}$ is an algebra norm if and only if $\alpha\|\cdot\|_{1} \leq \alpha \lambda\|\cdot\|_{2}$ or equivalently $R_{1,2} \leq \lambda$.

Proposition 2.9 Let $\|\cdot\|_{1}$ and $\|\cdot\|_{2}$ be two norms on $\mathbb{C}^{n}$ and $0 \neq \alpha, \beta \in \mathbb{C}$. Define $\|\cdot\|_{\alpha}$ and $\|\cdot\|_{\beta}$ on $\mathbb{C}^{n}$ by $\|x\|_{\alpha}=\|\alpha x\|_{1}$ and $\|x\|_{\beta}=\|\beta x\|_{2}$, respectively. Then $\|\cdot\|_{\alpha}$ and $\|\cdot\|_{\beta}$ are two norms on $\mathbb{C}^{n}$ and $\|\cdot\|_{\alpha, \beta}=\left|\frac{\beta}{\alpha}\right|\|\cdot\|_{1,2}$.

Proof. We have $\|A\|_{\alpha, \beta}=\max \left\{\|A x\|_{\beta}:\|x\|_{\alpha}=1\right\}=\max \left\{\|\beta A x\|_{2}:\|\alpha x\|_{1}=1\right\}=$ $\left|\frac{\beta}{\alpha}\right| \max \left\{\|A y\|_{2}:\|y\|_{1}=1\right\}=\left|\frac{\beta}{\alpha}\right|\|A\|_{1,2} \cdot$

The preceding proposition leads us ti give the following definition:

Definition 2.10 Let $\left(\|\cdot\|_{1},\|\cdot\|_{2}\right)$ and $\left(\|\cdot\|_{3},\|\cdot\|_{4}\right)$ be two pairs of norms on $\mathbb{C}^{n}$. We say that $\left(\|\cdot\|_{1},\|\cdot\|_{2}\right)$ is generalized induced congruent (gi-congeruent) to $\left(\|\cdot\|_{3},\|\cdot\|_{4}\right)$ and we write $\left(\|\cdot\|_{1},\|\cdot\|_{2}\right) \equiv_{g i}\left(\|\cdot\|_{3},\|\cdot\|_{4}\right)$ if $\|\cdot\|_{1,2}=\gamma\|\cdot\|_{3,4}$ for some $0<\gamma \in \mathbb{R}$.

Clearly $\equiv_{g i}$ is an equivalence relation. We denote by $\left[\left(\|\cdot\|_{1},\|\cdot\|_{2}\right)\right]_{g i}$ the equivalence class of $\left(\|\cdot\|_{1},\|\cdot\|_{2}\right)$. Proposition 2.9 shows that for each $0<\alpha, \beta \in \mathbb{R}$ we have $\left(\alpha\|\cdot\|_{1}, \beta\|\cdot\|_{2}\right) \equiv_{g i}$ $\left(\|\cdot\|_{1},\|\cdot\|_{2}\right)$. Indeed, we have the following result:

Theorem 2.11 For each pair $\left(\|\cdot\|_{1},\|\cdot\|_{2}\right)$ of norms on $\mathbb{C}^{n}$ we have $\left[\left(\|\cdot\|_{1},\|\cdot\|_{2}\right)\right]_{g i}=\left\{\left(\alpha\|\cdot\|_{1}, \beta\|\cdot\|_{2}\right)\right.$ : $0<\alpha, \beta \in \mathbb{R}\}$.

We can extend the above method to find some other norms on $M_{n}$ which are not necessarily gi-congruent to a given pair $\left(\|\cdot\|_{1},\|\cdot\|_{2}\right)$ :

Proposition 2.12 Let $\left(\|\cdot\|_{1},\|\cdot\|_{2}\right)$ be a pair of norms on $\mathbb{C}^{n}$ and $K, L \in M_{n}$ be two invertible matrices. Define \|\|$_{K}$ and \|\|$_{L}$ and $\mathbb{C}^{n}$ by $\|x\|_{K}=\|K x\|_{1}$ and $\|x\|_{L}=\|L x\|_{2}$. Then \|\|$_{K}$ and \|\|$_{L}$ are norms on $\mathbb{C}^{n}$ and $\|A\|_{K, L}=\left\|L A K^{-1}\right\|_{1,2}$. 
Proof. Clear and see also Lemma 3.1 of [4].

Remark 2.13 Note that the case $K=\alpha I$ and $L=\beta I$ gives Proposition 2.9.

\section{References}

[1] Belitskiǔ, G. R.; Lyubich, Yu. I. Matrix norms and their applications. Translated from the Russian by A. Iacob. Operator Theory: Advances and Applications, 36. Birkhuser Verlag, Basel, 1988.

[2] Bhatia, Rajendra. Matrix analysis. Graduate Texts in Mathematics, 169. SpringerVerlag, New York, 1997.

[3] Horn, Roger A.; Johnson, Charles R. Matrix analysis. Cambridge University Press, Cambridge, 1985.

[4] Li, Chi-Kwong; Tsing, Nam-Kiu; Zhang, Fuzhen. Norm hull of vectors and matrices. Linear Algebra Appl. 257 (1997), 1-27.

[5] Rudin, Walter. Real and Complex Analysis, McGraw-Hill, Singapore, 1987. 